\title{
Quantum Threshold Voltage Modeling of Short Channel Quad Gate Silicon Nanowire Transistor
}

\author{
P Rakesh Kumar and Santanu Mahapatra
}

\begin{abstract}
In this work, a physically based analytical quantum linear threshold voltage model for short channel quad gate metal oxide semiconductor field effect transistors is developed. The proposed model, which is suitable for circuit simulation, is based on the analytical solution of three-dimensional Poisson and two-dimensional Schrödinger equation. Proposed model is fully validated against the professional numerical device simulator for a wide range of device geometries and also used to analyse the effect of geometry variation on the threshold voltage.
\end{abstract}

Index Terms-Compact modeling, Quantum effects, Multi gate transistors, CMOS.

\section{INTRODUCTION}

Q UAD gate metal oxide field effect transistors has attracted much attention for downscaling CMOS (complementary metal oxide semiconductor) technology upto $10 \mathrm{~nm}$ channel length due to its maximum gate control over the channel and high current drive capability [1]. In such transistors the short channel effect (SCE) is controlled by the device geometry, and hence an undoped (or, lightly doped) ultra-thin body is used to sustain the channel. Since the quantization of electron energy cannot be ignored in such ultra-thin body devices, it is extremely important to consider quantum effects in their threshold voltage models. To the best of our knowledge, quantum threshold voltage model for short channel devices have not yet been reported apart from the recent long channel model, which is implicit in nature [2]. In this work, we propose a physically based closed form quantum linear threshold voltage model, which is applicable for ultra-thin and ultra-short channel quad gate devices and does not contain any unphysical fitting parameter. The 3D Poisson equation and 2D Schrödinger equation (with square potential well approximation) is solved in the weak inversion region to obtain the threshold voltage model. It is shown that electron distribution in energy subbands in the quad gate Metal Oxide Semiconductor Field Effect Transistor (MOSFET) is quite different from the bulk and double gate MOSFET. The proposed models are validated against the numerical device simulator Atlas [3] for different device architecture. The effect of effective mass and geometry variation on the threshold voltage is also studied using the proposed model.

This work is funded by Department of science and Technology (DST), India under Grant SR/S3/EECE/047/2008

P.Rakesh kumar (email:prakesh@cedt.iisc.ernet.in) and Santanu Mahapatra (email:santanu@cedt.iisc.ernet.in) are with Nano-Scale Device Research Laboratory, Centre for Electronics Design and Technology, Indian Institute of Science, Bangalore-560012, India.
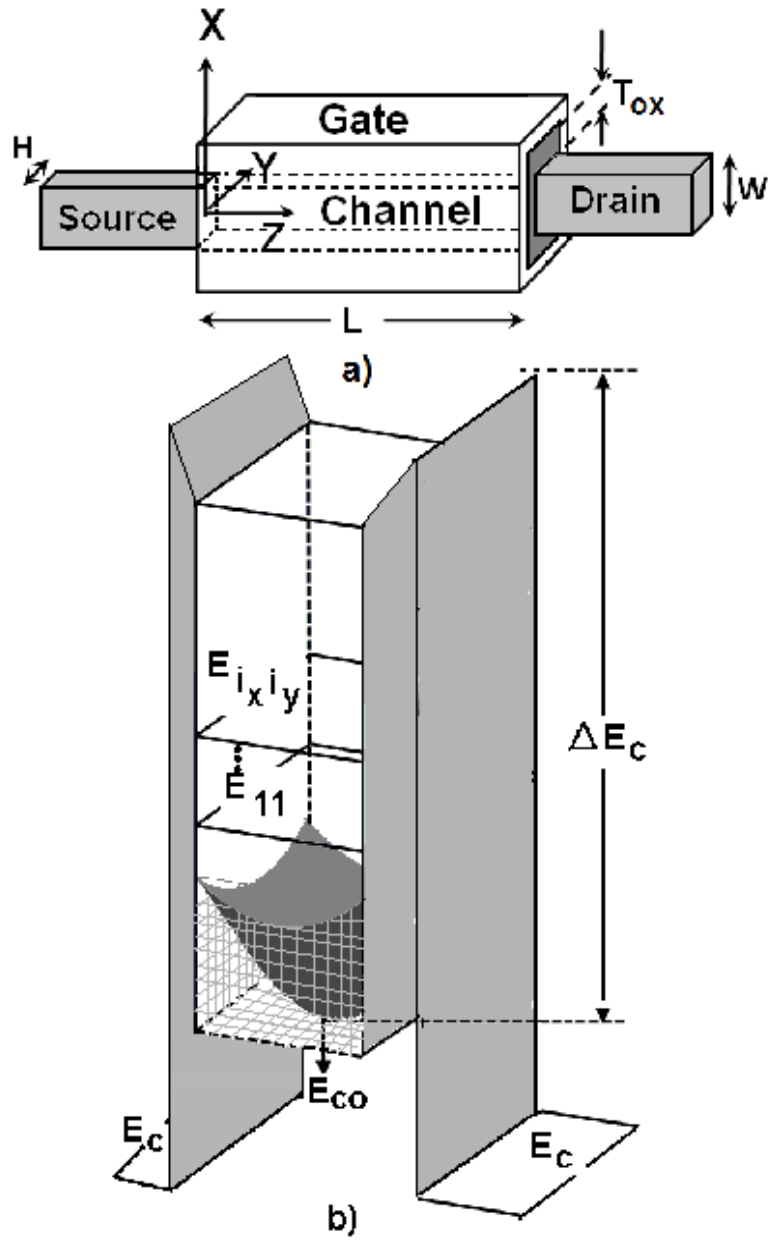

Figure 1. a) Schematic of quad gate transistor b) Band diagram perpendicular to the gate

\section{Model Development}

\section{A. Potential Modeling}

In ultra thin devices, the quantization of electron energy due to structural confinement becomes significant. Hence the Poisson-Schrödinger equations have to be solved consistently to obtain potential distribution and inversion charge density. However, in the weak inversion regime, one can approximate the Poisson equation as the Laplace equation by ignoring the inversion charge density, and hence decouple the two equations. In the development of threshold voltage models we have also taken the parabolic band approximation. We have used mid gap metals for the gates. Figure 1 shows the 
schematic diagram of an undoped (or lightly doped) quad gate MOSFET. The same voltage is applied to all the four gates. In both the insulator and silicon region the Poisson (Laplace) equation becomes

$$
\frac{\delta^{2} \Phi}{\delta x^{2}}+\frac{\delta^{2} \Phi}{\delta y^{2}}+\frac{\delta^{2} \Phi}{\delta z^{2}}=0
$$

with the boundary conditions

$$
\begin{array}{r}
\Phi\left(x,-H / 2-T_{o x}, z\right)=V_{g} \\
\Phi\left(-W / 2-T_{o x}, y, z\right)=V_{g} \\
\left.\frac{\delta \phi}{\delta x}\right|_{x=0}=0 \\
\left.\frac{\delta \phi}{\delta y}\right|_{y=0}=0 \\
\Phi(x, y, 0)=V_{b i} \\
\Phi(x, y, L)=V_{b i}+V_{d s} \simeq V_{b i} \text { for low } V_{d s}
\end{array}
$$

Here $\Phi$ is the potential, $V_{b i}$ is the built-in potential of a $n^{+}-i$ diode, $V_{g}$ is the gate potential and $V_{d s}$ is the drain to source voltage, $H$ and $W$ are the height and width of the silicon film, $T_{o x}$ is the gate oxide thickness and $L$ is the channel length respectively. Three dimensional numerical simulation results show that in the insulator region the potential can be approximated as a linear function. We further neglect the corner effects. The potential inside the insulator region can then be expressed by linear interpolation as

$$
\begin{array}{r}
\Phi(x, y, 0)=\frac{V_{g}-V_{b i}}{T_{o x}}\left(x-\frac{W}{2}\right)+V_{b i} \\
\text { for } \frac{W}{2}<x<\frac{W}{2}+T_{o x} \text { and } 0<y<\frac{H}{2} \\
\Phi(x, y, 0)=\frac{V_{g}-V_{b i}}{T_{o x}}\left(y-\frac{H}{2}\right)+V_{b i} \\
\text { for } 0<x<\frac{W}{2} \text { and } \frac{H}{2}<y<\frac{H}{2}+T_{o x}
\end{array}
$$

Using superposition, the potential can be written as

$$
\Phi(x, y, z)=v(x, y)+u_{L}(x, y, z)+u_{R}(x, y, z)
$$

Here $v(x, y)$ is the long channel potential which satisfy the boundary conditions (2) and (3). $v(x, y)$ should also satisfy the dielectric boundary conditions, namely $\epsilon(\delta v / \delta x)$ and $v(x, y)$ are continuous at $x=W / 2$ and $\epsilon(\delta v / \delta y)$ and $v(x, y)$ are continuous at $y=H / 2$. For quad gate MOSFET where all the gates have same voltage we have $v(x, y)=V_{g}$. Potentials $u_{L}(x, y, z)$ and $u_{R}(x, y, z)$ capture SCE and satifies the boundary conditions (6) and (7). Potentials $u_{L}(x, y, z)$ and $u_{R}(x, y, z)$ can be written as

$u_{L}=\sum_{n} \sum_{m} a_{T n m} \sin \left(\lambda_{n}\left(x-\frac{W}{2}-T_{o x}\right)\right) \cos \left(\mu_{m} y\right) \times$ $\frac{\sinh \left(\sigma_{n m}(L-z)\right)}{\sinh \left(\sigma_{n m} L\right)}$ for $\frac{W}{2}<x<\frac{W}{2}+T_{o x}$ and $0<y<\frac{H}{2}$

$$
\begin{aligned}
& u_{L}=\sum_{n} \sum_{m} a_{b n m} \cos \left(\lambda_{n} x\right) \sin \left(\mu_{m}\left(y-\frac{H}{2}-T_{o x}\right)\right) \times \\
& \frac{\sinh \left(\sigma_{n m}(L-z)\right)}{\sinh \left(\sigma_{n m} L\right)} \text { for } 0<x<\frac{W}{2} \text { and } \frac{H}{2}<y<\frac{H}{2}+T_{o x}
\end{aligned}
$$

$$
\begin{gathered}
u_{L}=\sum_{n} \sum_{m} a_{n m} \cos \left(\lambda_{n} x\right) \cos \left(\mu_{m} y\right) \times \\
\frac{\sinh \left(\sigma_{n m}(L-z)\right)}{\sinh \left(\sigma_{n m} L\right)} \text { for } 0<x<\frac{W}{2} \text { and } 0<y<\frac{H}{2} \\
u_{R}=\sum_{n} \sum_{m} b_{T n m} \sin \left(\lambda_{n}\left(x-\frac{W}{2}-T_{o x}\right)\right) \cos \left(\mu_{m} y\right) \times \\
\frac{\sinh \left(\sigma_{n m} z\right)}{\sinh \left(\sigma_{n m} L\right)} \text { for } \frac{W}{2}<x<\frac{W}{2}+T_{o x} \text { and } 0<y<\frac{H}{2}
\end{gathered}
$$

$$
\begin{aligned}
u_{R}= & \sum_{n} \sum_{m} b_{b n m} \cos \left(\lambda_{n} x\right) \sin \left(\mu_{m}\left(y-\frac{H}{2}-T_{o x}\right)\right) \times \\
& \frac{\sinh \left(\sigma_{n m} z\right)}{\sinh \left(\sigma_{n m} L\right)} \text { for } 0<x<\frac{W}{2} \text { and } \frac{H}{2}<y<\frac{H}{2}+T_{o x}
\end{aligned}
$$

$$
\begin{gathered}
u_{R}=\sum_{n} \sum_{m} b_{n m} \cos \left(\lambda_{n} x\right) \cos \left(\mu_{m} y\right) \times \\
\frac{\sinh \left(\sigma_{n m} z\right)}{\sinh \left(\sigma_{n m} L\right)} \text { for } 0<x<\frac{W}{2} \text { and } 0<y<\frac{H}{2}
\end{gathered}
$$

Here $a_{T n m}, a_{b n m}, a_{n m}, b_{T n m}, b_{b n m}$ and $b_{n m}$ are the constants to be obtained from the boundary conditions (6) and (7). $\sigma_{n m}$ is given by the following expression

$$
\sigma_{n m}=\sqrt{\lambda_{n}^{2}+\mu_{m}^{2}}
$$

The Poisson equation requires $\Phi$ and $\epsilon(\delta \Phi / \delta x)$ be continuous in the $x$ direction and $\Phi$ and $\epsilon(\delta \Phi / \delta y)$ be continuous in the $y$ direction. The dielectric boundary conditions at the two silicon-insulator interfaces requires $u_{L}$ and $\epsilon\left(\delta u_{L} / \delta x\right)$ be continuous at $x=W / 2$ and $u_{L}$ and $\epsilon\left(\delta u_{L} / \delta y\right)$ be continuous at $y=H / 2$. Applying continuity in (11) and (13) along $x$ direction we get

$$
\begin{array}{r}
-a_{T n m} \sin \left(\lambda_{n} T_{o x}\right)=a_{n m} \cos \left(\frac{\lambda_{n} W}{2}\right) \\
a_{T n m} \epsilon_{o x} \lambda_{n} \cos \left(\lambda_{n} T_{o x}\right)=-a_{n m} \epsilon_{s i} \lambda_{n} \sin \left(\frac{\lambda_{n} W}{2}\right)
\end{array}
$$

Taking the ratio of the above two equations we get $\lambda_{n}$ from the following equation

$$
\epsilon_{s i} \tan \left(\lambda_{n} T_{o x}\right)-\epsilon_{o x} \cot \left(\frac{\lambda_{n} T}{2}\right)=0
$$


Similarly applying continuity in (12) and (13) along $y$ direction and taking their ratio we get a equation for $\mu_{m}$ as

$$
\epsilon_{s i} \tan \left(\mu_{m} T_{o x}\right)-\epsilon_{o x} \cot \left(\frac{\mu_{m} H}{2}\right)=0
$$

Note that $\lambda_{n}$ and $\mu_{m}$ depends only on the device parameters. Now $u_{L}$ can be rewritten as $s_{n m} U_{L n m}$ where

$$
\begin{array}{r}
u_{L n m}=\alpha_{n m} \sin \left(\lambda_{n}\left(x-\frac{W}{2}-T_{o x}\right)\right) \cos \left(\mu_{m} y\right) \times \\
\frac{\sinh \left(\sigma_{n m}(L-z)\right)}{\sinh \left(\sigma_{n m} L\right)} \text { for } \frac{W}{2}<x<\frac{W}{2}+T_{o x} \text { and } 0<y<\frac{H}{2}
\end{array}
$$

$$
\begin{gathered}
u_{L n m}=\beta_{n m} \cos \left(\lambda_{n} x\right) \sin \left(\mu_{m}\left(y-\frac{H}{2}-T_{o x}\right)\right) \times \\
\frac{\sinh \left(\sigma_{n m}(L-z)\right)}{\sinh \left(\sigma_{n m} L\right)} \text { for } 0<x<\frac{W}{2} \text { and } \frac{H}{2}<y<\frac{H}{2}+T_{o x}
\end{gathered}
$$

$$
\begin{array}{r}
u_{L n m}=\gamma_{n m} \cos \left(\lambda_{n} x\right) \cos \left(\mu_{m} y\right) \frac{\sinh \left(\sigma_{n m}(L-z)\right)}{\sinh \left(\sigma_{n m} L\right)} \\
\text { for } 0<x<\frac{W}{2} \text { and } 0<y<\frac{H}{2}
\end{array}
$$

Eigen functions $u_{L n m}$ as defined by (22)-(24) are not orthogonal to each other. In order to evaluate the coefficient $s_{n m}$ we need to construct a corresponding conjugate function set $g_{n}$. It turns out that $g_{n m}$ can be made from the same set of functions as in (22)-(24) by choosing different multipliers in different regions and constants $\alpha_{n m}, \beta_{n m}$ and $\gamma_{n m}$ appropriately. The Conjugate function set $g_{n m}$ is found as

$$
\begin{gathered}
g_{n m}=\frac{\epsilon_{o x}}{2 \epsilon_{s i}} \frac{\sin \left(\lambda_{n}\left(x-\frac{W}{2}-T_{o x}\right)\right) \cos \left(\mu_{m} y\right)}{\sin \left(\lambda_{n} T_{o x}\right) \cos \left(\frac{\mu_{m} H}{2}\right)} \text { for } \\
\frac{W}{2}<x<\frac{W}{2}+T_{o x} \text { and } 0<y<\frac{H}{2} \\
g_{n m}=\frac{\epsilon_{o x}}{2 \epsilon_{s i}} \frac{\cos \left(\lambda_{n} x\right) \sin \left(\mu_{m}\left(y-\frac{H}{2}-T_{o x}\right)\right)}{\cos \left(\frac{\lambda_{n} W}{2}\right) \sin \left(\mu_{m} T_{o x}\right)} \text { for } \\
0<x<\frac{W}{2} \text { and } \frac{H}{2}<y<\frac{H}{2}+T_{o x} \\
g_{n m}=-\frac{\cos \left(\lambda_{n} x\right) \cos \left(\mu_{m} y\right)}{\cos \left(\frac{\lambda_{n} W}{2}\right) \cos \left(\frac{\mu_{m} H}{2}\right)} \text { for } 0<x<\frac{W}{2} \\
\text { and } 0<y<\frac{H}{2}
\end{gathered}
$$

The constants $\alpha_{n m}, \beta_{n m}$ and $\gamma_{n m}$ are given as

$$
\begin{aligned}
\alpha_{n m} & =\cos \left(\frac{\lambda_{n} W}{2}\right) \sin \left(\mu_{m} T_{o x}\right) \\
\beta_{n m} & =\cos \left(\frac{\mu_{m} H}{2}\right) \sin \left(\lambda_{n} T_{o x}\right) \\
\gamma_{n m} & =-\sin \left(\lambda_{n} T_{o x}\right) \sin \left(\mu_{m} T_{o x}\right)
\end{aligned}
$$

Similarly $u_{R}$ can be written as $t_{n m} u_{R n m}$ where $u_{R n m}$ is given as

$$
\begin{aligned}
& u_{R n m}=\alpha_{n m} \sin \left(\lambda_{n}\left(x-\frac{W}{2}-T_{o x}\right)\right) \cos \left(\mu_{m} y\right) \times \\
& \frac{\sinh \left(\sigma_{n m} z\right)}{\sinh \left(\sigma_{n m} L\right)} \text { for } \frac{W}{2}<x<\frac{W}{2}+T_{o x} \text { and } 0<y<\frac{H}{2}
\end{aligned}
$$

$$
\begin{aligned}
& u_{R n m}=\beta_{n m} \cos \left(\lambda_{n} x\right) \sin \left(\mu_{m}\left(y-\frac{H}{2}-T_{o x}\right)\right) \times \\
& \frac{\sinh \left(\sigma_{n m} z\right)}{\sinh \left(\sigma_{n m} L\right)} \text { for } 0<x<\frac{W}{2} \text { and } \frac{H}{2}<y<\frac{H}{2}+T_{o x}
\end{aligned}
$$

$$
\begin{array}{r}
u_{R n m}=\gamma_{n m} \cos \left(\lambda_{n} x\right) \cos \left(\mu_{m} y\right) \frac{\sinh \left(\sigma_{n m} z\right)}{\sinh \left(\sigma_{n m} L\right)} \\
\text { for } 0<x<\frac{W}{2} \text { and } 0<y<\frac{H}{2}
\end{array}
$$

$u_{L n m}$ at $x=W / 2$ and $y=H / 2$ from (22) is given as

$$
\begin{array}{r}
u_{L n m}=-\alpha_{n m} \sin \left(\lambda_{n} T_{o x}\right) \cos \left(\mu_{m} \frac{H}{2}\right) \\
=-\sin \left(\mu_{m} T_{o x}\right) \sin \left(\lambda_{n} T_{o x}\right) \cos \left(\mu_{m} \frac{H}{2}\right) \cos \left(\lambda_{n} \frac{W}{2}\right)
\end{array}
$$

$u_{L n m}$ at $x=W / 2$ and $y=H / 2$ from (23) is given as

$$
\begin{array}{r}
u_{L n m}=-\beta_{n m} \sin \left(\mu_{m} T_{o x}\right) \cos \left(\lambda_{n} \frac{W}{2}\right) \\
=-\sin \left(\mu_{m} T_{o x}\right) \sin \left(\lambda_{n} T_{o x}\right) \cos \left(\mu_{m} \frac{H}{2}\right) \cos \left(\lambda_{n} \frac{W}{2}\right)
\end{array}
$$

$u_{L n m}$ at $x=W / 2$ and $y=H / 2$ from (24) is given as

$$
\begin{array}{r}
u_{L n m}=-\gamma_{n m} \cos \left(\mu_{m} \frac{H}{2}\right) \cos \left(\lambda_{n} \frac{W}{2}\right) \\
=-\sin \left(\mu_{m} T_{o x}\right) \sin \left(\lambda_{n} T_{o x}\right) \cos \left(\mu_{m} \frac{H}{2}\right) \cos \left(\lambda_{n} \frac{W}{2}\right)
\end{array}
$$

Since Potential is given by $\sum_{n} \sum_{m} s_{s n m} u_{L n m}$ and from (34)(36) it is seen that $u_{L n m}$ gives the same value at the corner, potential is continuous at the corner. By multiplying (22)(24) with the corresponding conjugate functions (25)-(27) and integrating, the coefficients $s_{n m}$ can be obtained as

$$
\begin{gathered}
s_{n m}=\frac{\int_{0}^{H+T_{o x}} \int_{0}^{W+T_{o x}}(\Phi(x, y, 0)-v(x, y)) g_{n m}(x, y) d x d y}{\int_{0}^{H+T_{o x}} \int_{0}^{W+T_{o x}} u_{L n m}(x, y, 0) g_{n m}(x, y) d x d y} \\
=\left(V_{g}-V_{b i}\right) \frac{\Omega_{n m}}{\zeta_{n m}}
\end{gathered}
$$

Similarly $t_{n m}$ can be obtained as

$$
\begin{gathered}
t_{n m}=\frac{\int_{0}^{H+T_{o x}} \int_{0}^{W+T_{o x}}(\Phi(x, y, L)-v(x, y)) g_{n m}(x, y) d x d y}{\int_{0}^{H+T_{o x}} \int_{0}^{W+T_{o x}} u_{R n m}(x, y, L) g_{n m}(x, y) d x d y} \\
=\left(V_{g}-V_{b i}-V_{d s}\right) \frac{\Omega_{n m}}{\zeta_{n m}}
\end{gathered}
$$


where $\Omega_{n m}$ and $\zeta_{n m}$ are given by the following equations

$$
\begin{array}{r}
\Omega_{n m}=\frac{\epsilon_{o x} \mu_{m} \tan \left(\frac{\mu_{m} H}{2}\right)+\epsilon_{o x} \lambda_{n} \tan \left(\frac{\lambda_{n} W}{2}\right)}{2 \epsilon_{s i} T_{o x} \mu_{m}^{2} \lambda_{n}^{2}} \\
-\frac{2}{\mu_{m} \lambda_{n}} \tan \left(\frac{\mu_{m} H}{2}\right) \tan \left(\frac{\lambda_{n} W}{2}\right) \\
\zeta_{n m}=\frac{\sin \left(\mu_{m} T_{o x}\right)}{16 \cos \left(\frac{\mu_{m} H}{2}\right)}\left(H+\frac{\sin \left(\mu_{m} H\right)}{\mu_{m}}\right) \times \\
\left(\frac{\epsilon_{o x} T_{o x} \cos \left(\frac{\lambda_{n} W}{2}\right)}{\epsilon_{s i} \sin \left(\lambda_{n} T_{o x}\right)}+\frac{W \sin \left(\lambda_{n} T_{o x}\right)}{2 \cos \left(\frac{\lambda_{n} W}{2}\right)}\right)+ \\
\frac{\sin \left(\lambda_{n} T_{o x}\right)}{16 \cos \left(\frac{\lambda_{n} W}{2}\right)}\left(W+\frac{\sin \left(\lambda_{n} W\right)}{\lambda_{n}}\right) \times \\
\left(\frac{\epsilon_{o x} T_{o x} \cos \left(\frac{\mu_{m} H}{2}\right)}{\epsilon_{s i} \sin \left(\mu_{m} T_{o x}\right)}+\frac{H \sin \left(\mu_{m} T_{o x}\right)}{2 \cos \left(\frac{\mu_{m} H}{2}\right)}\right)
\end{array}
$$

Using (10) potential in the silicon region can be written as

$$
\begin{aligned}
\Phi(x, y, z)= & V_{g}+\left(V_{g}-V_{b i}\right) \sum_{n} \sum_{m} c_{n m} \sinh \left(\sigma_{n m}(L-z)\right) \\
& +\left(V_{g}-V_{b i}-V_{d s}\right) \sum_{n} \sum_{m} \sinh \left(\sigma_{n m} z\right)
\end{aligned}
$$

where $c_{n m}$ is given by

$$
c_{n m}(x, y)=-\frac{\Omega_{n m} \gamma_{n m}}{\zeta_{n m}} \frac{\cos \left(\lambda_{n} x\right) \cos \left(\mu_{m} y\right)}{\sinh \left(\sigma_{n m} L\right)}
$$

\section{B. Classical Threshold Voltage Modeling}

Threshold voltage for undoped body devices is defined as the gate voltage when the integrated charge at the virtual cathode becomes equal to the critical charge $\left(Q_{T}\right)$. It is found from the numerical simulation that only the first series term in (41) is sufficient to predict the potential at virtual cathode and hence in the further analysis only the first term is used. Classical integrated charge is obtained from (41) as

$$
Q=\int_{-H / 2}^{H / 2} \int_{-W / 2}^{W / 2} q n_{i} e^{\left(\frac{\Phi}{U_{T}}\right)} d x d y
$$

Now, as $\Phi$ is a very complicated function of $x$ and $y$, the above integration cannot be evaluated analytically. So the above integration can be approximated as:

$$
Q \approx W H q n_{i} e^{\left(\frac{\Phi\left(\frac{3 W}{14}, \frac{3 H}{14, y}, z_{c}\right)}{U_{T}}\right)}
$$

Here $U_{T}$ is the thermal voltage, $n_{i}$ is the intrinsic carrier concentration, $z_{c}$ is the virtual cathode position which is $L / 2$ for low $V_{d s}$ and $q$ is the elementary charge. Using (44) and (41) the classical threshold voltage model is obtained as

$$
V_{T C}=\frac{U_{T} \ln \left(\frac{Q_{T}}{W H q n i}\right)+2 V_{b i} c_{11}\left(\frac{3 W}{14}, \frac{3 H}{14}\right) \sinh \left(\frac{\sigma_{11} L}{2}\right)}{1+2 c_{11}\left(\frac{3 W}{14}, \frac{3 H}{14}\right) \sinh \left(\frac{\sigma_{11} L}{2}\right)}
$$
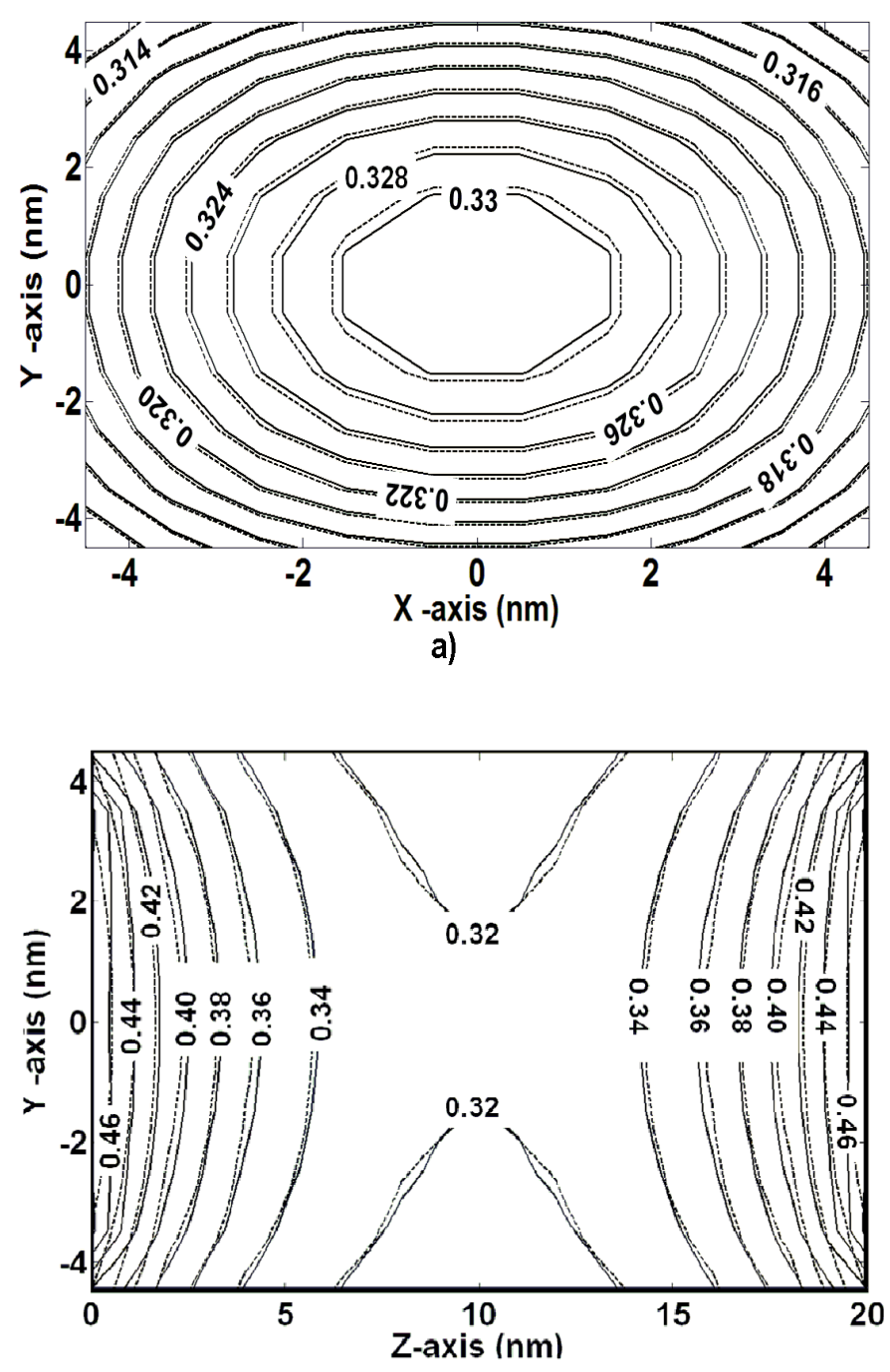

b)

Figure 2. Constant electrostatic potential contours based on the analytical solution (Solid curves) for quad gate transistor compared with 3D simulation results from Atlas (dashed lines). Here $L=20 \mathrm{~nm}, H=9 \mathrm{~nm}, W=9 \mathrm{~nm}$ and $V_{g}=0.3 \mathrm{~V}$ a) $X-Y$ plane at the virtual cathode $\left(z_{c}=L / 2\right.$ b) $Y-Z$ plane at $X=0$.

\section{Quantum Threshold Voltage Modeling}

The Potential distribution obtained in (41) is quasi parabolic in nature in $x$ and $y$ direction (see Fig.1b). Hence it is very difficult to solve Schrödinger equation with the potential expression given in (41). Therefore in this work, we approximate the actual potential well as the square potential well as shown by the checked lines in Fig. 1. In this square potential well, the bottom represents the minima of conduction band energy $\left(E_{c o}\right)$ at the body centre position $\left(x=0, y=0, z=z_{c}\right)$ and can be given as

$$
E_{c o}=\frac{E_{g}}{2}-q \Phi\left(0,0, z_{c}\right)
$$


Using the above value of potential energy (46), the Schrödinger equation becomes

$$
\frac{\hbar^{2}}{2 m_{x}} \frac{\delta^{2} \Psi}{\delta x^{2}}+\frac{\hbar^{2}}{2 m_{y}} \frac{\delta^{2} \Psi}{\delta y^{2}}+\left(E-E_{c o}\right) \Psi=0
$$

Equation (47) can be solved by standard variable separable technique[5] and its solution $(\Psi)$ and $(E)$ is given as

$$
\begin{array}{r}
\Psi_{i_{x} i_{y}}(x, y)=\sqrt{\left(\frac{4}{H W}\right)} \sin \left(\frac{i_{x} \pi\left(x-\frac{l_{x}}{2}\right)}{l_{x}}\right) \times \\
\sin \left(\frac{i_{y} \pi y-\frac{l_{y}}{2}}{l_{y}}\right) \\
E_{i_{x} i_{y}}=E_{c o}+\frac{\hbar^{2} \pi^{2}}{2}\left[\frac{1}{m_{x}}\left(\frac{i_{x}}{l_{x}}\right)^{2}+\frac{1}{m_{y}}\left(\frac{i_{y}}{l_{y}}\right)^{2}\right]
\end{array}
$$

Here $\hbar=h /(2 \pi), h$ is the planks constant, $\Psi$ is the wave function and $E$ is the energy of the electron wave. In silicon there are six ellipsoidal valleys with $m_{t}$ and $m_{l}$ as the transverse and longitudinal effective masses and $i_{x}$ and $i_{y}$ are the positive natural numbers. In (48) and (49), the masses $\left(m_{x}\right.$ and $\left.m_{y}\right)$ and the lengths $l_{x}$ and $l_{y}$ take different values depending on the direction of quantization. For example,If masses and dimensions of the film are $m_{l}$ and $m_{t}$ and $W$ and $H$ along the quantization direction, then in (48) and (49) $m_{x}$ and $m_{y}$ will assume the mass of $m_{t}$ and $m_{l}$ and $l_{x}$ and $l_{y}$ will assume the lengths as $W$ and $H$ respectively. The energy reaches a minimum for a maximum mass (49). For Silicon with six energy valleys we have thus two lower energy valleys, two middle energy valleys and two higher energy valleys respectively. In a special case of equal height and width of the film, the two lower energy valleys and two middle energy valleys merge producing four lower energy valleys and two higher energy valleys. Thus the charge per unit length per valley is given by

$$
Q=\sum_{i_{x}} \sum_{i_{y}} q \int_{E_{i_{x} i_{y}}}^{\infty} N_{1 D} f(E) d(E)
$$

Using $N_{1 D}$ as the one dimensional density of states and $f(E)$ as the Fermi-dirac function (50) leads to

$$
Q=q \sum_{i_{x}} \sum_{i_{y}} \sqrt{\left(\frac{m_{z}}{2 \pi \hbar^{2}}\right)} \int_{E_{i_{x} i_{y}}}^{\infty} \frac{\left(E-E_{i_{x} i_{y}}\right)^{\frac{-1}{2}}}{1+\exp \left(\frac{E-E_{F}}{k T}\right)} d E
$$

Here $m_{z}$ is the mass of that valley which is perpendicular to the direction of quantization.The charge equation (51) is difficult to solve. However in the weak inversion regime the Fermi level is found to be much below the conduction band energy. Hence using Bolzmann statistics the integrated charge could be approximated as

$$
\begin{array}{r}
Q=q \sqrt{\left(\frac{m_{z}}{2 \pi \hbar^{2}}\right)} \sum_{i_{x}} \sum_{i_{y}} \int_{E_{i_{x} i_{y}}}^{\infty} \frac{\exp \left(\frac{E_{F}-E}{k T}\right)}{\sqrt{\left(E-E_{i_{x} i_{y}}\right)}} d E \\
=q \sum_{i_{x}} \sum_{i_{y}} \sqrt{\frac{m_{z} k T}{2 \hbar^{2}}} \exp \left(\frac{E_{F}-E_{i_{x} i_{y}}}{k T}\right)
\end{array}
$$

Using (49) and (52), the total integrated charge at virtual cathode $(z=L / 2)$ is given by

$$
\begin{array}{r}
Q=\sum_{i_{x}} \sum_{i_{y}} q \sqrt{\frac{2 k T m_{t}}{\hbar^{2}}} \exp \left(-\frac{E_{c o}+\kappa\left(i_{x}, i_{y}\right)}{k T}\right)+ \\
\sum_{i_{x}} \sum_{i_{y}} q \sqrt{\frac{2 k T m_{t}}{\hbar^{2}}} \exp \left(-\frac{E_{c o}+\varpi\left(i_{x}, i_{y}\right)}{k T}\right)+ \\
\sum_{i_{x}} \sum_{i_{y}} q \sqrt{\frac{2 k T m_{t}}{\hbar^{2}}} \exp \left(-\frac{E_{c o}+\omega\left(i_{x}, i_{y}\right)}{k T}\right)
\end{array}
$$

where

$$
\begin{aligned}
& \kappa\left(i_{x}, i_{y}\right)=\frac{\hbar^{2} \pi^{2}}{2}\left[\frac{1}{m_{l}}\left(\frac{i_{x}}{W}\right)^{2}+\frac{1}{m_{t}}\left(\frac{i_{y}}{H}\right)^{2}\right] \\
& \varpi\left(i_{x}, i_{y}\right)=\frac{\hbar^{2} \pi^{2}}{2}\left[\frac{1}{m_{t}}\left(\frac{i_{x}}{W}\right)^{2}+\frac{1}{m_{l}}\left(\frac{i_{y}}{H}\right)^{2}\right] \\
& \omega\left(i_{x}, i_{y}\right)=\frac{\hbar^{2} \pi^{2}}{2}\left[\frac{1}{m_{t}}\left(\frac{i_{x}}{W}\right)^{2}+\frac{1}{m_{t}}\left(\frac{i_{y}}{H}\right)^{2}\right]
\end{aligned}
$$

However it is seen from simulation that only one energy level is sufficient to predict the charge accurately. Hence only one energy level is used in the further anaylsis.

Using (46) and (53) and the same definition of $Q_{T}$ the quantum threshold voltage model becomes

$$
V_{T Q}=\frac{\frac{E_{g}}{2 q}+\frac{k T}{q} \ln \left(\frac{Q_{T}}{\rho}\right)+2 V_{b i} c_{11}(0,0) \sinh \left(\frac{\sigma_{11} L}{2}\right)}{1+2 c_{11}(0,0) \sinh \left(\frac{\sigma_{11} L}{2}\right)}
$$

where $\rho$ is given by

$$
\begin{gathered}
\rho=q \sqrt{\frac{2 k T m_{t}}{\hbar^{2}}}\left(\exp \left(-\frac{\kappa(1,1)}{k T}\right)+\exp \left(-\frac{\varpi(1,1)}{k T}\right)\right) \\
+q \sqrt{\frac{2 k T m_{l}}{\hbar^{2}}} \exp \left(-\frac{\omega(1,1)}{k T}\right)
\end{gathered}
$$

The increase in the threshold voltage due to quantum effects is obtained from (45) and (57) and is given by

$$
\Delta V_{T}=V_{T Q}-V_{T C}
$$

\section{Results AND Discussion}

Figure 2 shows the constant electrostatic potential contours of a quad gate transistor. We have used only one series term $(n=1, m=1)$ and 25 series terms $(n=5, m=5)$ in (10) for obtaining $2 \mathrm{a}$ and $2 \mathrm{~b}$ respectively. It is seen from the plot that only one series term is sufficient to predict the potential at virtual cathode but one needs several terms to predict the potential close to source and drain region. Thus the assumption of linear potential variation at the insulator boundaries is valid. 


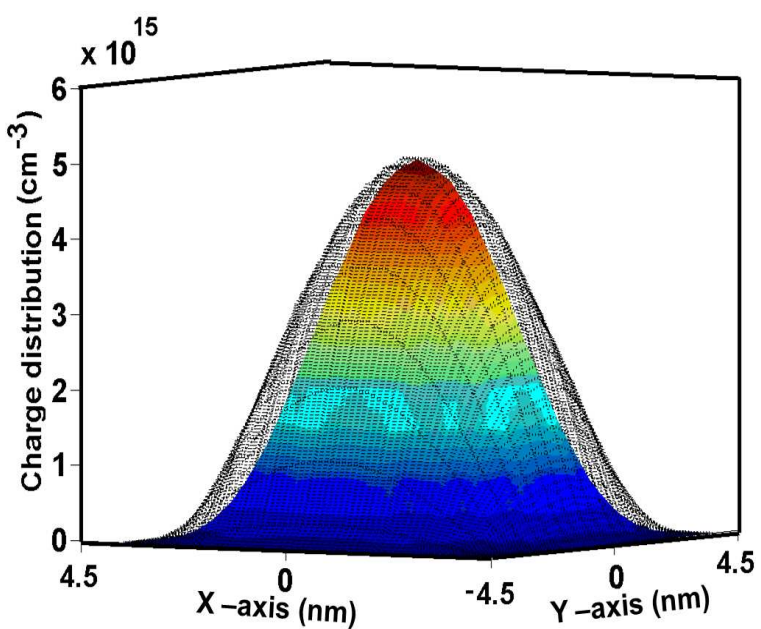

Figure 3. Charge distribution plot at virtual cathode for QG at $V_{g}=0.3 \mathrm{~V}$. Here $H=9 \mathrm{~nm}, W=9 \mathrm{~nm}$ and $L=20 \mathrm{~nm}$. Symbols represent Atlas simulation and lines represent the model.

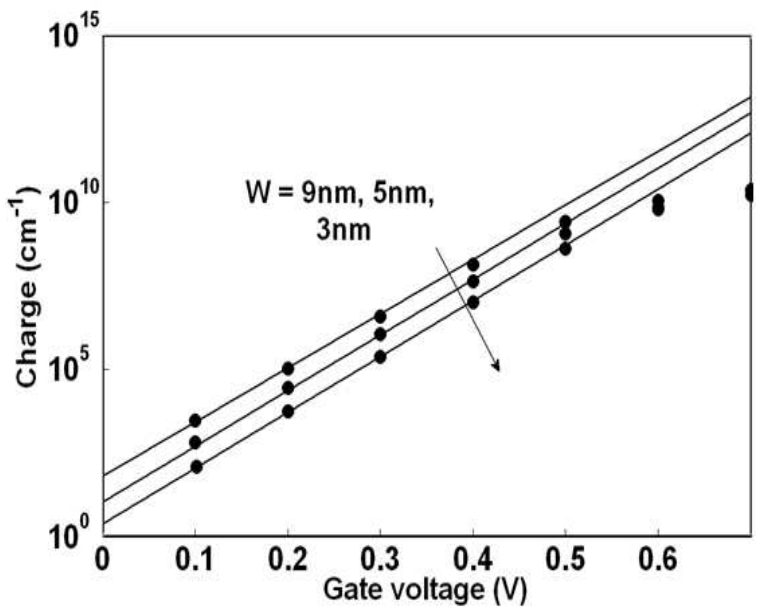

Figure 4. Variation of quantum integrated charge at virtual cathode with gate voltage for different film width for $H=9 \mathrm{~nm}$ and $L=20 \mathrm{~nm}$. Symbols and lines represent the Atlas simulation and model respectively.

It is also observed that although we neglect the insulator corners, the potential is continuous at the corners. Figure 3 shows the quantum charge distribution plot at virtual cathode position at gate voltage $0.3 \mathrm{~V}$. Color represents the model and the line represents the 3D Atlas simulation results. Figure 4 represents the variation of total quantum integrated charge with gate voltage. It is seen from the figure that the quantum threshold voltage increase with decrease in film thickness. This is due to increase in energy quantization with decrease in film thickness. Equation (53) is used to obtain the integrated charge with only one energy level and one series term.

Figure 5 shows the variation of the quantum threshold voltage with width and height of the film at channel length of $20 \mathrm{~nm}$. Figure 6 shows the variation of quantum and classical threshold voltage with film height for different channel lengths at film width of $9 \mathrm{~nm}$. The vertical spacing between the lines in Fig.6 gives the SCE. It is observed that the SCE decreases slightly with energy quantization. This is due to increase in

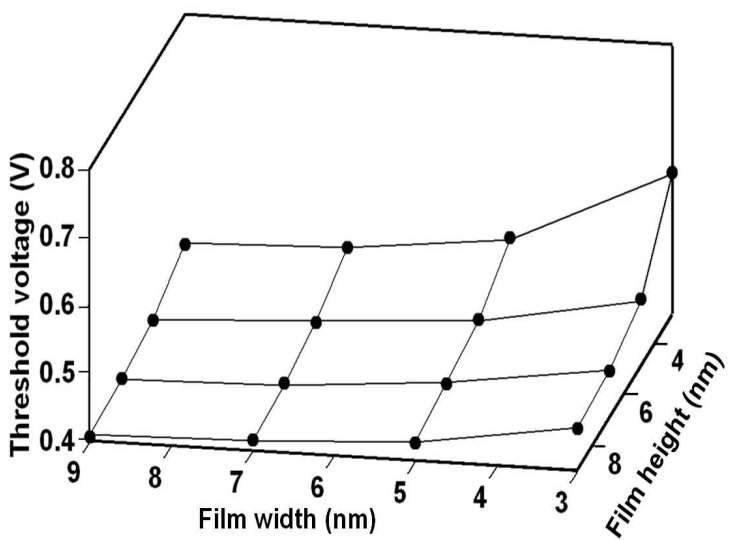

Figure 5. Variation of quantum threshold voltage with film height and width for channel length $(L=20 \mathrm{~nm})$. Symbols and lines represents the Atlas simulation and model respectively.

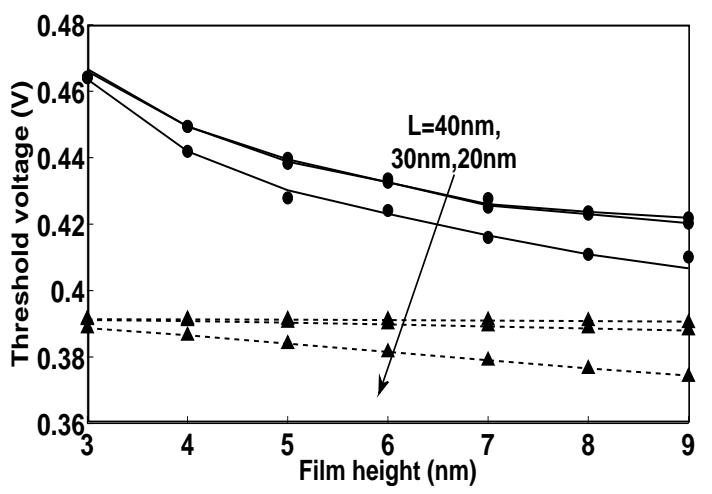

Figure 6. Variation of threshold voltage with film height for different channel lengths for $W=9 \mathrm{~nm}$. Line and circle symbol represents the quantum threshold voltage model and the quantum Atlas simulation.Dashed line and triangle symbol represents the classical threshold voltage model and the Atlas simulation

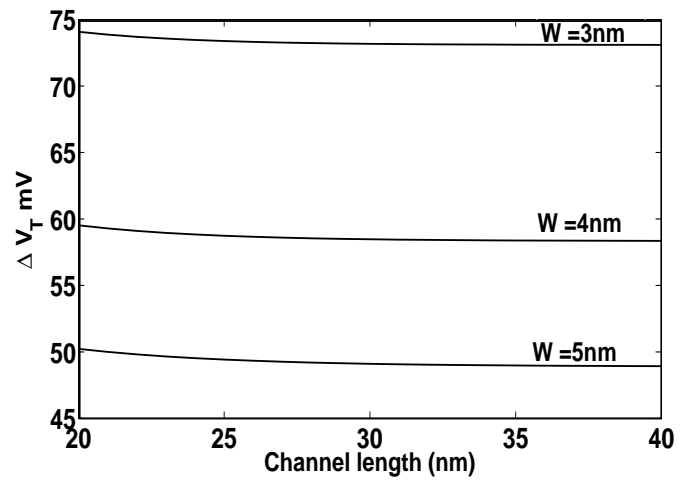

Figure 7. Variation of $\Delta V_{T}$ with channel length for variuos widths ( $H=$ $9 \mathrm{~nm})$. 


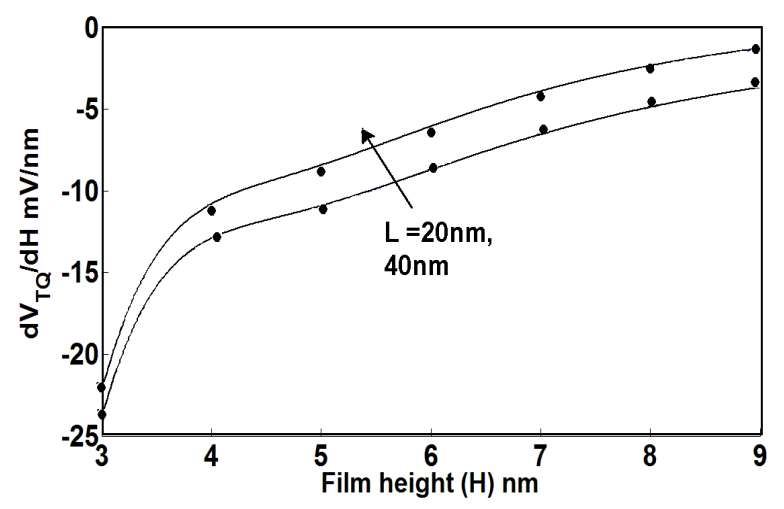

Figure 8. Variation of $d V_{T Q} / d H$ with film height for different channel lengths. Line and Symbols indicates the variation at at $W=8 \mathrm{~nm}$ and $W=$ $9 \mathrm{~nm}$.

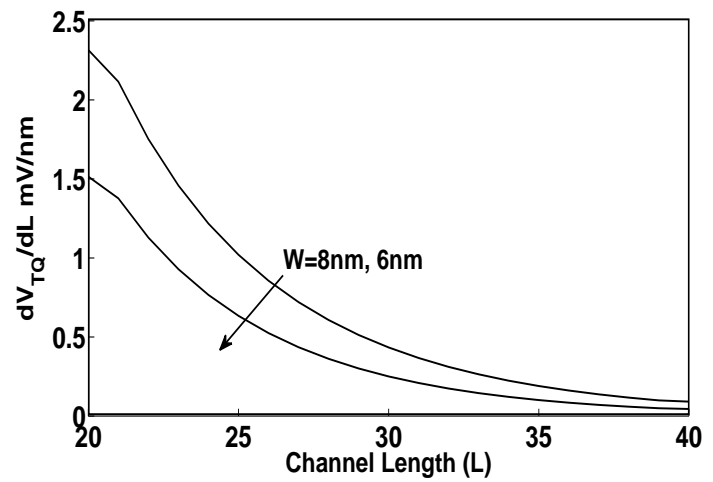

Figure 9. Variation of $d V_{T Q} / d L$ with channel Lengths for different film widths at $H=9 \mathrm{~nm}$.

the effective band gap of silicon because of quantum effects [8]. The effect of confinement $\left(\Delta V_{T}\right)$, as obtained from (59) is shown in Fig.7 as a function of device dimensions.

In literatures no standard value is found for the threshold charge $\left(Q_{T}\right)$. Some authors [7] have equated it with the thermal charge. However we have found from the numerical simulation that the threshold voltage obtained by using the thermal charge sometimes (depending on the device geometry)

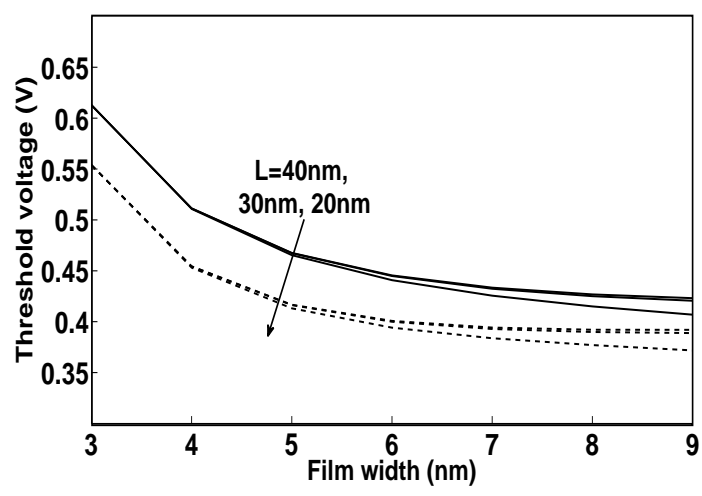

Figure 10. Variation of threshold voltage with channel Lengths for different film widths at $H=W$. Line and dashed line represents the quantum threshold voltage with bulk effective mass and modified effective mass $m^{*}$. lies in the strong inversion regime and thus over predict the threshold voltage. In this work we first extract the threshold voltage from classical simulation of $I_{d}-V_{g}$ characteristics by linear interpolation method for wide range of device architectures. Corresponding to the extracted threshold voltage, integrated charge at virtual cathode is computed for each device and an average charge is taken as the threshold charge which is $5 \times 10^{24} \mathrm{qW} \mathrm{Hem}^{-1}$.

Figures 8 and 9 show the variation of first derivative of quantum threshold voltage with film height and length respectively. A quad gate transistor is symmetric about width and height. Hence the derivative of quantum threshold voltage with respect to channel width also remains almost same as Fig. 8. These figures are obtained by numerically computing the derivatives of quantum threshold voltage obtained from the proposed model. It is seen from the Fig. 8 that the variation is high at lower film height compared to higher values which is due to the quantum confinement effect. The effect of variation of device geometries on the quantum threshold voltage could be obtained by using the chain rule as follows

$$
\begin{array}{r}
\frac{\Delta V_{T Q}}{V_{T Q}}=\frac{W}{V_{T Q}} \frac{\delta V_{T Q}}{\delta W} \frac{\Delta W}{W}+\frac{H}{V_{T Q}} \frac{\delta V_{T Q}}{\delta H} \frac{\Delta H}{H}+ \\
\frac{L}{V_{T Q}} \frac{\delta V_{T Q}}{\delta L} \frac{\Delta L}{L}
\end{array}
$$

Now for example if there is $5 \%$ increase in width of the transistor due to process variation for the geometry $W=9 \mathrm{~nm}$, $H=8 \mathrm{~nm}$ and $L=20 \mathrm{~nm}$, using the derivative values from Figs. 8 and 9 we get the following value for $\Delta V_{T Q}$ as:

$$
\frac{\Delta V_{T Q}}{V_{T Q}}=-0.0048 \frac{\Delta W}{W}-0.0032 \frac{\Delta H}{H}+0.002316 \frac{\Delta L}{L}
$$

If we assume zero variation in channel length, the variation in threshold voltage can be made zero if film height decreases by an amount of $7.5 \%$. In this way in a quad gate MOSFET, the effect of variation in one dimension could be compansated by properly tuning the other dimension.

So far in the discussion we assumed the value of the effective masses to be equal to the value of bulk Silicon effective masses. However the values of effective masses changes with the device dimensions. For a cilindrical body Silicon nanowire transistor, having a diameter $d$, the effective mass could be formulated as a function of $d$ by following equation [9]:

$$
m_{l, t}^{*}(d)=m_{l, t}^{*}(\infty)\left(1+\frac{a_{l, t}}{d}+\frac{b_{l, t}}{d^{2}}\right)
$$

where $a_{l, t}$ and $b_{l, t}$ are the fitting parameters having the values $a_{l}=0, a_{t}=0.68, b_{l}=0.28$ and $b_{t}=0.87 \cdot m_{l, t}^{*}(\infty)$ denotes the effective mass in bulk Silicon. In this work we approximated the value of equivalent nanowire diameter to be equal to $(W+H) / 2$. In Figure 10 the lines shows the variation of threshold voltage if we use the constant bulk effective mass and dashed lines shows the variation of threshold voltage if we use effective mass obtained from (62). It is seen from the figure that the threshold voltage is low when we consider the effective mass dependence on device geometry rather than assuming it to be a constant. 


\section{CONCLUSIONS}

A physically based analytical linear quantum threshold voltage model for a quad gate has been developed and verified a against professional numerical simulator. The proposed model, which is based on the solution of the Poisson and Schrödinger equations does not use any fitting parameters and is capable of predicting the threshold voltage for ultra short channel channel and ultra thin body devices.

\section{REFERENCES}

[1] J.P.Colinge, "FinFETs and Other Multi-Gate Transistors", Springer, 2007.

[2] Munteanu et. al, "Compact modeling of the threshold voltage in silicon nanowire MOSFET including 2D-quantum confinement effects", Molecular simulation, vol 31, pp 839-845, Oct 2005.

[3] Atlas user manual,version 5.14.0.R

[4] Xiaoping Liang and Yuan Taur, "A 2-D Analytical solution for SCEs in DG MOSFEts", IEEE Transactions on Electron Devices, vol 51, no 8 pp 1384-1391, 2004.

[5] Erwin Kreyszig, "Advanced Engineering Mathematics",John Wiley and sons, 2005

[6] David J.Griffith, "Introduction to quantum mechanics" , AddisonWesley, 2004.

[7] Munteanu et. al, "Quantum short channel compact model for the threshold voltage in double gate MOSFETs with high-permittivity gate dielectrics", Journal of non-crystalline solids, vol 351, pp 1911-1918, 2005.

[8] M. J. van Dort, P. H. Woerlee and A. J. Walker, "A simple model for quantisation effects in heavily-doped silicon MOSFETs at inversion conditions", Solid state electronics, vol 37, no 3, pp 411-414, 1994.

[9] E. Gnani, S. Reggiani, M Rudan and G Baccarani "Effects of the BandStructure Modification in Silicon Nanowires with Small Diameters", Solid state device research conference, pp 170-173, 2006.

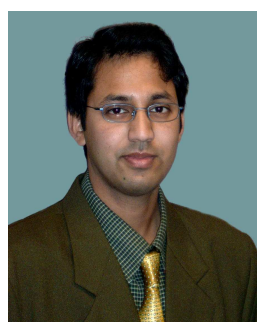

Santanu Mahapatra (M'08) received the B.E. degree in electronics and telecommunication from Jadavpur University, Kolkata, India, in 1999, the M.Tech. degree in electrical engineering with specialization in microelectronics from the Indian Institute of Technology (IIT), Kanpur, India, in 2001, and the Ph.D. degree in electrical engineering, from the Swiss Federal Institute of Technology Lausanne (EPFL), Lausanne, Switzerland, in 2005. In his $\mathrm{Ph} . \mathrm{D}$. he worked on compact modeling of Single Electron Transistors and their hybridization with

CMOS.

Since 2005, he has been an Assistant Professor at the Centre for Electronics Design and Technology (CEDT), Indian Institute of Science (IISc), Bangalore, India. He is the founder of the Nano Scale Device Research Laboratory at the CEDT, in 2006, where his team is engaged in research on compact modeling and simulation of emerging nanotechnologies and advanced CMOS devices. He provides consultancy to the device reliability group of Cypress Semiconductor, Bangalore. He is the author and coauthor of several papers published in international journals and refereed conferences. He is also the author of the book "Hybrid CMOS Single Electron Transistor Device and Circuit Design" (Artech House, 2006). His current research interests include device reliability,multigate transistors, tunnel field-effect transistors, singleelectron transistors, and CMOS-nano hybridization.

Dr. Mahapatra received the Best Paper Award in the International Semiconductor Conference (CAS), Romania, in 2003. He is also the recipient of IBM Faculty Award in 2007, Microsoft India Research Outstanding Faculty Award, in 2007 and the associateship of Indian Academy of Science in 2009.

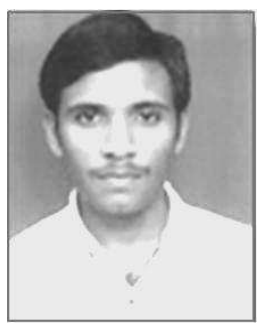

P. Rakesh kumar received the B.Tech degree in electrical engineering from National Institute of Technology (NIT), Kurukshetra, India, in 2007. He is currently working toward the M.Sc. (Engg.) degree at Indian Institute of Science, Bangalore, India His current research interests include RF Analog circuits, compact modeling, simulation of semiconductor devices. 